\title{
Power law relationship between parameters of earthquakes and precursory electrical phenomena revisited II
}

\author{
E. Dologlou \\ Solid State Section, Department of Physics, University of Athens, Panepistimiopolis, Zografos, 15784 Athens, Greece
}

Received: 15 April 2010 - Revised: 4 June 2010 - Accepted: 7 June 2010 - Published: 1 July 2010

\begin{abstract}
The credibility of the power law relation, reported by Dologlou (2009) between the stress drop of an earthquake and the lead time of the preceded Seismic Electric Signal, SES, has been checked through additional new data from 9 June 2008 to 7 April 2010. Three earthquakes with $M_{\mathrm{w}} \geq 5.5$ have been found in Greece during this period. A critical exponent $\alpha$ (e.g. 0.328) has been obtained which compares very well with the one reported $(\alpha=0.332)$ by Dologlou (2009). The stability of this exponent might imply that critical dynamic processes, of mechanical (earthquakes) and also of electromagnetic (SES) sense dominate the pre focal area when the SES signal of the impending earthquake is emitted.
\end{abstract}

\section{Introduction}

The last 3 decades low frequency $(<1 \mathrm{~Hz})$ variations of the earth's electric field, known as seismic electric signals (SES), have been monitored at different stations in Greece and were found to precede large earthquakes (Varotsos and Alexopoulos, 1984a, b; Varotsos and Lazaridou, 1991; Varotsos et al., 1993). A fundamental feature of the SES signal is the lead time $\Delta t$, which is the time difference between the detection of the SES and the occurrence of the associated earthquake and varies from some hours to some weeks (Varotsos and Alexopoulos, 1984 a, b; Varotsos et al., 1996).

Recently, the introduction of a new time domain, termed natural time (Varotsos et al., 2002; 2003a, b, 2005), allowed the identification of the time of the impending earthquake with accuracy of the order of one week (Varotsos et al., 2006a, b). As for the epicentral area it is estimated on the basis of the so called SES selectivity which states that a SES station can be sensitive to some specific seismic areas

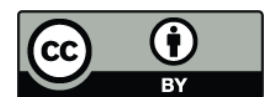

Correspondence to: E. Dologlou (edologl@phys.uoa.gr) while remains inactive to some others even at closer distances (Varotsos and Lazaridou, 1991; Varotsos et al., 1993; Sarlis et al., 1999)

A brief description of the physical mechanism of the SES generation is given below: The various materials that exist in the Earth's crust contain intrinsic lattice defects (Varotsos and Alexopoulos, 1978, 1979, 1984c) e.g. anion Frenkel in alkaline earth fluorides (Varotsos, 1976, 2007), vacancies in metals (Varotsos and Alexopoulos, 1982), Schotcky defects in ionic crystals. Beyond these defects, however, extrinsic also defects are formed for charge compensation reasons (Kostopoulos et al., 1975; Varotsos et al., 1978) particularly in ionic solids (Varotsos and Alexopoulos, 1981) when are doped with aliovalent impurities. A portion of these defects are attracted by the nearby impurities, thus forming electric dipoles the orientation of which can change by means of a defect migration according to the relation

$\tau=(\lambda \nu)^{-1} \exp (g / k T)$

where $T$ denotes the temperature, $\lambda$ the number of jump paths accessible to jumping species with an attempt frequency $v$ and $g$ the Gibbs energy for the (re)orientation process. Pressure affects the value of $g$ (Varotsos, 1977; Varotsos and Alexopoulos, 1978) as expressed by

$v=(\mathrm{d} g / \mathrm{d} P)_{T}$

where $v$ denotes the migration or the activation volume in general (Varotsos et al., 1998) Thus, if $v<0$ (Varotsos and Alexopoulos, 1980), an increase of pressure results in a decrease of the relaxation time $\tau$. When the pressure reaches a critical value $P=P_{\mathrm{cr}}$ a transient current, arising from a cooperative (re)orientation of dipoles, is emitted with a relaxation time $\tau\left(P_{\mathrm{cr}}\right)$ following relation:

$\frac{b v}{k T}=-\frac{1}{\tau\left(P_{\mathrm{cr}}\right)}$

where $b(=\mathrm{d} P / \mathrm{d} t)$ is the rate of the gradual pressure increase. 
Let us now examine the dynamical processes which are developing in the prefocal area. Before an earthquake, the preseismic volume is subjected to an increasing tectonic stress which affects the thermodynamic parameters of this migration (Varotsos, 1977) and may cause a gradual decrease of their relaxation time. When the stress reaches a critical value the dipoles in the rocks in the pre-seismic area, change orientation cooperatively and a transient signal (Varotsos and Alexopoulos, 1986; Varotsos, 2005) is emitted. This signal constitutes the SES activity and it is characterized by critical dynamics.

The amplitude $E=\Delta V / L$ of the SES, where $\Delta V$ is the potential difference between to points on the ground at a distance $L$ measured by two buried electrodes, is related to the magnitude $M$ of the forthcoming earthquake by the experimental formula:

$\log E=a M+b$

where $a \approx 0.3-0.4$ and $b$ is a site constant. Equation (4) is a power law relation that is reminiscent of the theory of critical phenomena (Varotsos and Alexopoulos, 1984 a, b; Varotsos, 2005).

Alternatively, Ishido and Mizutani (1981) suggested the electrokinetic effect for the SES generation, in terms of criticality, which leads to an exponent of 0.3-0.4 for critical phenomena (Surkov et al., 2002) while Sornette et al. (1989) consider that fracture processes are described by a power law relation with critical exponent values of 0.33 and 0.47 for 2 and 3 dimensions, respectively.

Possible relations between properties of earthquakes and characteristics of precursory SES signals have been investigated in a series of articles. Recently, (Dologlou, 2008, 2009) a power law relation between the stress drop of the earthquake, which is the difference between the stress state at a point on a fault before and after the occurrence of the earthquake (Kanamori and Anderson, 1975), and the lead time of the associated SES has been found with an exponent falling within the range of values for critical phenomena.

In this work we check the validity of this relation by means of additional new data and we examine the degree of stability of the critical exponent value.

\section{Data and analysis}

In a previous paper (Dologlou, 2009) we referred to large shallow (depth $<40 \mathrm{~km}$ ) earthquakes in Greece for which Brune's stress drop (Brune, 1970, 1971) values were either reported or derived by using a special technique based on the dimensions of the aftershock area and seismic moment (Kiratzi et al., 1991). Here, we deal with the largest events that occurred recently in Greece, i.e., after the Andravida case (on 8 June 2008). Three earthquakes with $M_{\mathrm{w}} \geq 5.5$ were found in the area $(36-41) \mathrm{N},(19-25) \mathrm{E}$ from 9 June 2008 to 6 April 2010; the first earthquake occurred in Ionian sea on
16 February 2009 at 23:16 UTC with $M_{\mathrm{w}}=5.5$ and epicenter 37.13N, 20.78E (event no. 17 in Table 1) and was preceded by a SES activity recorded at PIR station (Fig. 1a) on 12 December 2008 with a lead time $\Delta t=66$ days; the second earthquake with $M_{\mathrm{w}}=5.8$ took place on 3 November 2009 at $05: 25 \mathrm{UTC}$ with epicenter $37.5 \mathrm{~N}, 20.49 \mathrm{E}$ (event no. 18 in Table 1) and was preceded by a SES activity detected at PIR station (Fig. 1b) on 24 October 2009 with a lead time $\Delta t=10$ days. At last, on 18 January 2010 at 15:56 UTC a third earthquake with $M_{\mathrm{w}}=5.5$ (event no. 19 in Table 1) occurred in Central Greece $(38.39 \mathrm{~N} 21.90 \mathrm{E})$, close to the town of Nafpaktos, and caused moderate damage in the village of Efpalion (Fig. 1c). This event was also preceded by a SES activity recorded at PAT station on 11 November 2009 with a lead time $\Delta t=68$ days.

The Brune's stress drop values for these earthquakes were calculated through the formula of Hanks and Wyss (1972):

$\Delta \sigma_{\mathrm{B}}=0.44 M_{\mathrm{o}} / r^{3}$

where $M_{O}$ is the seismic moment and $\mathrm{r}$ the radius for a circular fault derived from $P$ and $S$ teleseismic waves displacement spectra. The estimation of the radius $r$, was obtained by the application of the aftershock area technique (Kiratzi, 1991) as it is described in details by Dologlou (2009). The definition of the dimensions of the aftershock area requires a rich aftershock sequence which was not in all cases available by USGS. Thus, in order to overcome this obstacle and enrich the number of aftershocks, we combined the USGS data with additional ones reported by the European Mediterranean Seismological Center, EMSC (http://www.emsc-csem.org) which by being closer to Greece is able to locate the small magnitude events $\left(M_{\mathrm{w}}>3\right)$. All aftershocks of the three main shocks with $M_{\mathrm{w}}>3$ reported either by USGS or EMSC for the periods, 16 February 2009 (23:17) to 30 March 2009, 3 November 2009 (05:26) to 31 December 2009, and 18 January 2010 (15:57) to 28 February 2010, respectively, are shown in separate maps in Fig. 1. In each map, the black star denotes the main shock, the beach ball represents its CMT focal mechanism (http://earthquake.usgs.gov/ earthquakes/eqarchives/sopar/). For events 18 and 19 the focal mechanisms were taken from NOA (National Observatory of Athens) because there are not available USGS yet. At last, black square shows the location of the SES detection station. The aftershock area $S=L \times W$, where $L$ is the length and $W$ the width in $\mathrm{km}$, is well defined from the distribution of aftershocks. The radius $r$ is calculated from the equation $S=\pi r^{2}$. Concerning the seismic moment $M_{0}$, two slightly different values are reported for each event which are given in Tables 2, 3, and 4, respectively, along with their mean values which were used in Eq. (5) for the calculation of the stress drop. Table 1 is the updated version of the table reported by Dologlou (2009) including the 3 new earthquakes (events no. 17, 18, and 19). It shows all 19 earthquakes with available stress drop values and precursory SES signals in Greece from 1981 to 2010, along with their dates, epicentres, 
Table 1. The updated version of the table reported by Dologlou (2009) including 3 new earthquakes in Ionian Sea and Central Greece (events no. 17, 18, and 19). All 19 earthquakes are presented with available stress drop values and precursory SES signals in Greece from 1981 to 2010, along with their dates, epicentres, depths, moment magnitudes $M_{\mathrm{W}}$, stress drop values $\left(\Delta \sigma_{\mathrm{B}}\right)$, SES lead times $\Delta t$ and mechanism type (strike-slip, normal or thrust). Events are numbered in chronological order and references for the stress drop values are given in the last column.

\begin{tabular}{|c|c|c|c|c|c|c|c|c|c|c|c|c|}
\hline$n$ & date & $\mathrm{H}$ & MIN & $\mathrm{S}$ & LAT & LONG & $\begin{array}{l}\text { Depth } \\
(\mathrm{km})\end{array}$ & $M_{\mathrm{W}}$ & $\begin{array}{l}\Delta \sigma_{\mathrm{B}} \\
\text { bars }\end{array}$ & $\begin{array}{l}\text { SES } \\
\Delta t \\
\text { (days) }\end{array}$ & $\begin{array}{l}\text { Source } \\
\text { mechanism }\end{array}$ & References \\
\hline 1 & 19 Dec 1981 & 14 & 10 & 50.7 & 39.24 & 25.23 & 12 & 6.8 & 9.01 & 0.3 & strike - slip & Dologlou (2008) \\
\hline 2 & 18 Jan 1982 & 19 & 27 & 24.5 & 40 & 24.32 & 6 & 6.6 & 10.5 & 0.3 & strike - slip & Dologlou (2008) \\
\hline 3 & 17 Jan 1983 & 12 & 41 & 29 & 38.09 & 20.19 & 10 & 6.9 & 14.0 & 1.8 & strike + thrust & Stavrakakis and Blionas (1990) \\
\hline 4 & 13 Sep 1986 & 17 & 24 & 34 & 37.03 & 22.2 & 15 & 5.9 & 5.0 & 5 & normal & Papazachos et al. (1988) \\
\hline 5 & 16 Oct 1988 & 12 & 34 & 6 & 37.95 & 20.90 & 29 & 5.9 & 2.53 & 17.5 & strike-slip & Dologlou (2009) \\
\hline 6 & 4 May 1995 & 0 & 34 & 11 & 40.54 & 23.63 & 15 & 5.4 & 2.5 & 28.5 & normal & Chouliaras-Stavrakakis (1997) \\
\hline 7 & 13 May 1995 & 8 & 47 & 15 & 40.16 & 21.67 & 15 & 6.5 & 6.3 & 25.5 & normal & Chouliaras-Stavrakakis (1997) \\
\hline 8 & 15 Jun 1995 & 0 & 15 & 56 & 38.1 & 22.46 & 15 & 6.5 & 2.9 & 46 & normal & Chouliaras-Stavrakakis (1997) \\
\hline 9 & 18 Nov 1997 & 14 & 7 & 53 & 37.33 & 20.84 & 22.9 & 6.6 & 1.42 & 45 & strike slip & Dologlou (2009) \\
\hline 10 & 7 Sep 1999 & 11 & 56 & 56 & 37.97 & 23.6 & 15 & 6 & 3.0 & 6 & normal & Stavrakakis-Chouliaras (2002) \\
\hline 11 & 26 Jul 2001 & 0 & 21 & 44 & 38.96 & 24.29 & 15 & 6.5 & 9.0 & 130 & strike - slip & Benetatos et al. (2002) \\
\hline 12 & 14 Aug 2003 & 5 & 15 & 8 & 38.7 & 20.67 & 15 & 6.3 & 8.0 & 6 & strike-slip & Papadimitriou (2007) \\
\hline 13 & 6 Jan 2008 & 5 & 14 & 20 & 37.22 & 22.69 & 75 & 6.2 & 9.0 & 60 & thrust + strike & Papadimitriou (2008) \\
\hline 14 & 4 Feb 2008 & 20 & 25 & 9.5 & 38.08 & 21.94 & 20 & 5 & 1.6 & 25 & strike-slip & Papadimitriou (2008) \\
\hline 15 & 14 Feb 2008 & 10 & 9 & 22.7 & 36.5 & 21.67 & 29 & 6.9 & 9.0 & 30 & thrust & Papadimitriou (2008) \\
\hline 16 & 8 Jun 2008 & 12 & 25 & 29.7 & 37.96 & 21.52 & 16 & 6.3 & 1.83 & 98 & strike-slip & Dologlou (2009) \\
\hline 17 & 16 Feb 2009 & 23 & 16 & 38.5 & 37.13 & 20.78 & 15 & 5.5 & 2.14 & 66 & strike - slip & see text \\
\hline 18 & 3 Nov 2009 & 5 & 25 & 8.1 & 37.50 & 20.49 & 10 & 5.8 & 3.11 & 10 & strike + thrust & see text \\
\hline 19 & 18 Jan 2010 & 15 & 56 & 9.0 & 38.40 & 21.96 & 1 & 5.5 & 2.1 & 68 & normal & see text \\
\hline
\end{tabular}
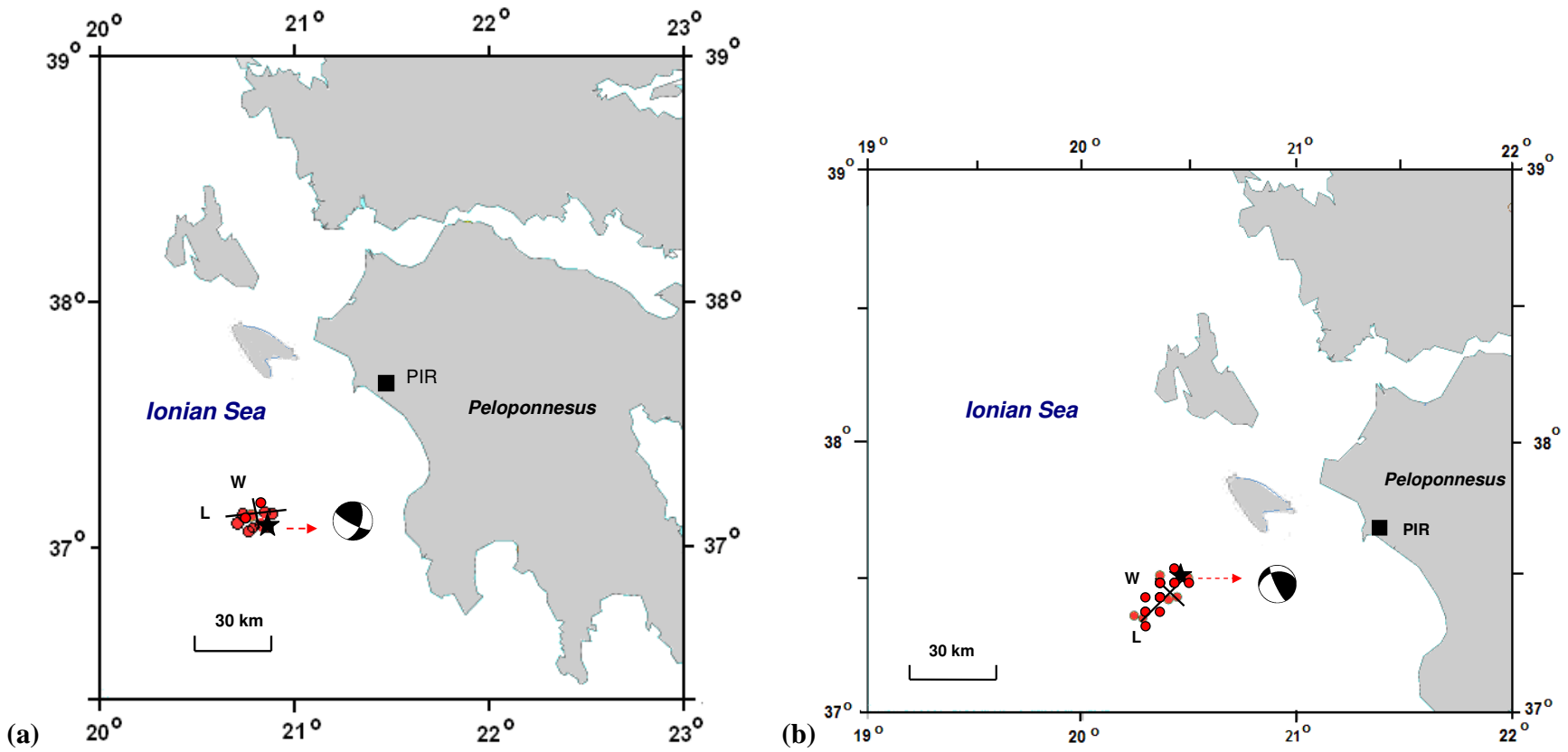

Fig. 1. Map of Western Greece, with the distribution (forming a cluster) of all reported by USGS or EMSC aftershocks with $M_{\mathrm{w}} \geq 3$ (red solid circles). (a) For event no. 17 (16 February 2009) for the period 16 February 2009 (23:17) to 30 March 2009. (b) For event no. 18 (3 November 2009) for the period 3 November 2009 (05:26) to 31 December 2009 and (c) for event no. 19 (18 January 2010 ) for the period 18 January $2010(15: 57)$ to 28 February 2010. The epicentres of the corresponding main shocks are presented by stars and their focal mechanisms by a lower hemisphere projection with black and white quadrants for compression and dilatation, respectively (beach ball). Representative aftershock areas of length $L$ and width $W$ for all cases are marked. Black squares denote the position of the SES stations that recorded the associated precursory signals. 
Table 2. The date, the magnitude $M_{\mathrm{W}}$ and the seismic moment $M_{\mathrm{O}}$ of event no. 17 along with the range of values in the dimensions of the aftershock area $\mathrm{L}$ and $\mathrm{W}$, the corresponding calculated values for $\Delta \sigma_{\mathrm{B}}$, the critical exponent $\alpha$ and the associated correlation coefficient $R$. Mean values are given in the last row.

\begin{tabular}{lccccccc}
\hline Date & $M_{\mathrm{W}}$ & $M_{\mathrm{O}}\left(10^{24}\right.$ dyn.cm $)$ & $\mathrm{L}(\mathrm{km})$ & $\mathrm{W}(\mathrm{km})$ & $\Delta \sigma_{\mathrm{B}}($ bars $)$ & $\alpha$ & $R$ \\
\hline 16 Feb 2009 & 5.5 & 2.2 & $18-20$ & $8-10$ & $3.1-1.9$ & $0.328-0.332$ & $0.80-0.83$ \\
& & 1.7 & $18-20$ & $8-10$ & $2.41-1.47$ & $0.324-0.341$ & $0.82-0.83$ \\
Mean value & & 1.95 & 19 & 9 & 2.14 & $0.331 \pm 0.01$ & $>0.80$ \\
\hline
\end{tabular}

Table 3. The date, the magnitude $M_{\mathrm{W}}$ and the seismic moment of event no. 18 along with the range of values in the dimensions of the aftershock area $\mathrm{L}$ and $\mathrm{W}$, the corresponding calculated values for $\Delta \sigma_{\mathrm{B}}$, the critical exponent $\alpha$ and the associated correlation coefficient $R$. Mean values are given in the last row.

\begin{tabular}{lccccccc}
\hline Date & $M_{\mathrm{W}}$ & $M_{\mathrm{O}}\left(10^{24}\right.$ dyn.cm $)$ & $\mathrm{L}(\mathrm{km})$ & $\mathrm{W}(\mathrm{km})$ & $\Delta \sigma_{\mathrm{B}}($ bars $)$ & $\alpha$ & $R$ \\
\hline 3 Nov 2009 & 5.8 & 8 & $26-28$ & $10-12$ & $4.67-3.18$ & $0.329-0.328$ & $0.82-0.80$ \\
& 5.7 & 5 & $26-28$ & $10-12$ & $2.92-1.98$ & $0.328-0.327$ & $0.82-0.80$ \\
Mean value & & 6.5 & 27 & 11 & 3.11 & $0.328 \pm 0.01$ & 0.81 \\
\hline
\end{tabular}

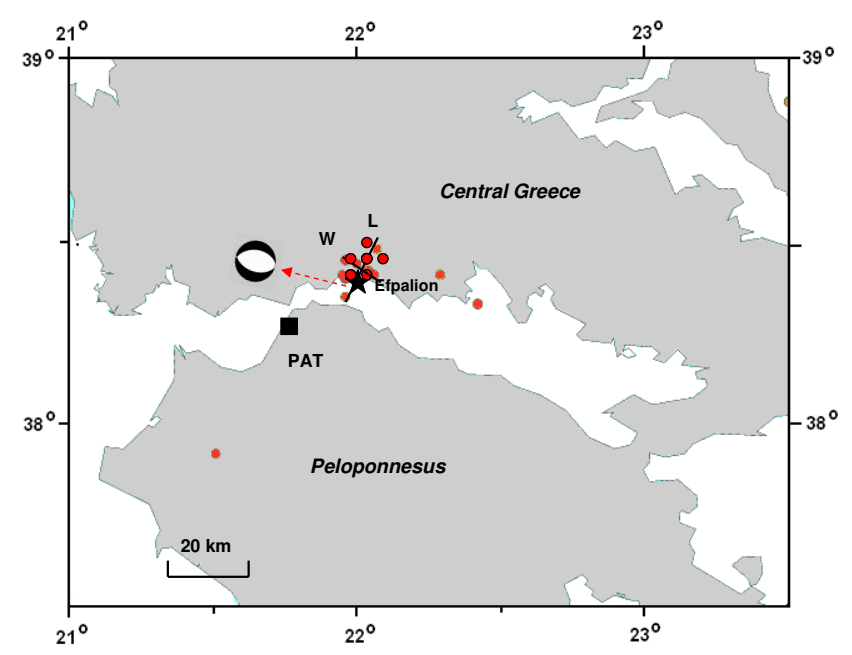

(c)

Fig. 1. Continued.

depths, moment magnitudes $M_{\mathrm{w}}$, stress drop values $\left(\Delta \sigma_{\mathrm{B}}\right)$, SES lead times $\Delta t$ and mechanism type (strike-slip, normal or thrust). Events are numbered in chronological order and references for the stress drop values are given in the last column. The lead times, $\Delta t$, for the events no. (1-16) are taken from the papers quoted by Dologlou (2009) while for the under study three recent events (no. 17, 18, and 19) from Varotsos et al. (2009a, b).

A power law relation with an exponent $\alpha$ is found between the stress drop $\Delta \sigma_{\mathrm{B}}$ values and the lead time $\Delta t$ of their precursory SES of all "non thrust" earthquake (Table 1). Event no. 11 is excluded from this relation for reasons explained in detail by Dologlou et al. (2008). This event had an unusual long lead time and occurred in a specific region of small crust thickness (Le Pichon et al., 1984) and high heat flow rate (Jongsma, 1974).

The date, the magnitude $M_{\mathrm{w}}$ and the seismic moment $M_{\mathrm{o}}$ along with the range of values in the dimensions $L$ and $W$ of the aftershock area, the corresponding calculated values for $\Delta \sigma_{\mathrm{B}}$, the critical exponent $\alpha$ and the associated correlation coefficient $R$ for events 17, 18, and 19 are presented in Tables 2, 3, and 4, respectively. Their mean values are also given in the last row.

\section{Discussion}

The following power law relation $\Delta \sigma_{\mathrm{B}}=8.18 \Delta t^{-0.329}$ between the stress drop $\Delta \sigma_{\mathrm{B}}$ values (in bars) of all "non thrust" earthquakes and the lead time $\Delta t$ (in s) of their precursory SES listed in Table 1 is obtained (see plot in Fig. 2) with an exponent $\alpha=0.329$ falling within the range value (0.3-0.4) empirically determined by Varotsos and Alexopoulos (1984). The dynamics of earthquakes of thrust mechanism which occur in zones of high stress accumulation, like subduction or collision zones, differ significantly from those of strike-slip or normal type mechanisms. Probably this fact could tentatively justify the observation that when thrust type earthquakes are included in the above power law relation the value of the exponent $\alpha$ is strongly violated.

The insertion of the additional new data of the three recent earthquakes in the above formula, that interconnects precursory SES characteristics and fracture properties (earthquakes) in the pre focal area, did not alter the value of the 
Table 4. The date, the magnitude $M_{\mathrm{W}}$ and the seismic moment of event no. 19 along with the range of values in the dimensions of the aftershock area $\mathrm{L}$ and $\mathrm{W}$, the corresponding calculated values for $\Delta \sigma_{\mathrm{B}}$, the critical exponent $\alpha$ and the associated correlation coefficient $R$. Mean values are given in the last row.

\begin{tabular}{lccccccc}
\hline Date & $M_{\mathrm{W}}$ & $M_{\mathrm{O}}\left(10^{24}\right.$ dyn.cm $)$ & $\mathrm{L}(\mathrm{km})$ & $\mathrm{W}(\mathrm{km})$ & $\Delta \sigma_{\mathrm{B}}(\mathrm{bars})$ & $\alpha$ & $R$ \\
\hline 18 Jan 2010 & 5.5 & 2.1 & $16-18$ & $9-11$ & $2.97-1.85$ & $0.314-0.332$ & $0.80-0.82$ \\
& 5.4 & 1.7 & $16-18$ & $9-11$ & $2.41-1.49$ & $0.322-0.341$ & $0.81-0.82$ \\
Mean value & & 1.9 & 17 & 10 & 2.1 & $0.328 \pm 0.02$ & 0.81 \\
\hline
\end{tabular}

exponent $\alpha$ which remains almost the same with the reported one ( $\alpha=0.332)$ by Dologlou (2009). This implies that this relation is quite robust and probably reveals that underlying critical dynamic processes govern the pre focal area when the SES of the forthcoming earthquake is emitted.

Considering now the range value of the dimension $L$ and $W$ for each event (Tables 2, 3, and 4) we notice that the exponent $\alpha$ spans between the values $0.32-0.34$ with a correlation coefficient $R>0.80$ in all cases and thus, remaining within the critical value range.

We will now discuss whether an interrelation between lead time and stress drop can be physically understood. The similar critical exponents in fracture processes (Sornette et al., 1989) and in the experimental Eq. (4) (Varotsos, 2005) probably implies that upon the emission of the SES, the pre focal area enters into a critical stage. As the tectonic stress increases, the pre-seismic volume evolves to the critical approach of the main-shock and long range correlations emerge in the area. The lead time $\Delta t$ between the emission of the SES and the earthquake is given by

$\Delta t=\left(P_{\mathrm{fr}}-P_{\mathrm{cr}}\right) / b$

(where $P_{\mathrm{fr}}$ is the critical stress for earthquake occurrence). Since $P_{\mathrm{fr}}>P_{\mathrm{cr}}$ is always positive, $\Delta t$ becomes shorter for rapid stress increase (larger $b$ ). During the last preparatory stage of a given earthquake when in general power law processes prevail, b value may change with time. From Eqs. (1) and (3) it can be shown (Varotsos et al., 1993) that

$\mathrm{d} P_{\mathrm{cr}} / \mathrm{d} b\left(P_{\mathrm{cr}}\right)=\tau\left(P_{\mathrm{cr}}\right)$

which reflects that $\mathrm{d} P_{\mathrm{cr}} / \mathrm{d} b\left(P_{\mathrm{cr}}\right)$ is always positive. Hence, for larger values of $b$, the critical pressure $P_{\text {cr }}$ becomes also larger. Then the numerator of the right hand side of Eq. (6) becomes smaller leading to the conclusion that an increase in the $b$ value causes a two-fold decrease in the lead time and vice versa. For different earthquakes, in the same geotectonic area, if $P_{\text {fr }}$ may approximately be assumed constant but the corresponding $b$ values (and therefore the $\mathrm{P}_{\mathrm{cr}}$ values) may be larger or smaller, reflecting - from Eq. (6) - smaller or larger $\Delta t$ values will result, respectively. The question is whether a similar situation can be expected for the interconnection between $\Delta t$ and the stress drop $\Delta \sigma$, because from physical point of view, stress drop is a quantity different

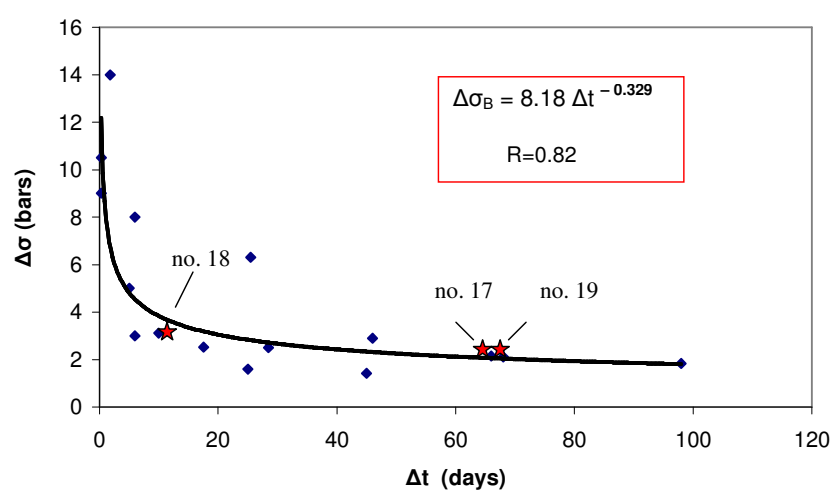

Fig. 2. The plot of the relation between the stress drop and the lead time of all "non thrust" earthquakes, which are listed in Table 1. Event no. 11 is excluded. The new events no. 17, 18, and 19 are marked with stars. The derived power law equation along with the corresponding correlation coefficient $\mathrm{R}$ are displayed on the top of the diagram.

from $\left(P_{\mathrm{fr}}-P_{\mathrm{cr}}\right)$. The precise value of this difference is still unknown, thus making difficult at this stage any attempt towards explaining why a power law of the form $\Delta \sigma_{\mathrm{B}} \propto \Delta t^{-\alpha}$ describes the experimental data although, such power laws are expected since we are dealing with critical processes.

\section{Conclusions}

New additional data from the three recent large earthquakes that occurred in Greece, from 9 June 2008 to the present, have been used and confirmed that the relation between the stress drop of an earthquake and the lead time of its precursory SES signal remains stable compared to a previous one found by Dologlou (2009). An exponent $\alpha=0.329$ was obtained which is also in striking agreement with the power law exponent that interrelates the SES amplitude and the earthquake magnitude experimentally found by Varotsos and Alexopoulos (1984a, b). The stability of this exponent might imply that critical dynamic processes, of mechanical (earthquakes) origin but also of electromagnetic (SES) sense govern the pre focal area when the SES signal of the impending earthquake is emitted. 
Edited by: M. E. Contadakis

Reviewed by: two anonymous referees

\section{References}

Benetatos, C., Roumelioti, Z., Kiratzi, A., and Melis, N.: Source parameters of the M 6.5 Skyros island (North Aegean Sea) earthquake of July 26, 2001, Ann. Geophys.-Italy, 45, 513-526, 2002.

Brune, J. : Tectonic stress and the spectra of seismic shear waves, J.Geophys. Res., 75, 4997-5009, 1970.

Brune, J. N.: Correction, J. Geophys. Res., 76, p. 5002, 1971.

Chouliaras, G. and Stavrakakis, G.: Seismic source parameters from a new dial-up seismological network in Greece, Pure Appl. Geophys., 150, 91-111, 1997.

Dologlou, E.: Power law relationship between parameters of earthquakes and precursory electrical phenomena, Nat. Hazards Earth Syst. Sci., 8, 977-983, doi:10.5194/nhess-8-977-2008, 2008.

Dologlou, E.: Power law relationship between parameters of earthquakes and precursory electrical phenomena revisited, Nat. Hazards Earth Syst. Sci., 9, 17-23, doi:10.5194/nhess-9-17-2009, 2009.

Dologlou, E., Hadjicontis, V., and Mavromatou, C.: Electrical precursors of earthquakes in Aegean Sea during the last decade (1997-2007), Nat. Hazards Earth Syst. Sci., 8, 123-128, doi:10.5194/nhess-8-123-2008, 2008.

Hanks, T. and Wyss, M.: The use of body wave spectra in the determination of seismic source parameters, B. Seismol. Soc. Am., 62, 561-589, 1972.

Ishido, T. and Mizutani, H.: Experimental and theoretical basis of electrokinetic phenomena in rock-water systems and its applications to geophysics, J. Geophys. Res., 86, 1763-1775, 1981.

Jongsma, D.: Heat Flow in the Aegean Sea, Geophys. J. Int., 37(3), 337-346, 1974.

Kanamori, H. and Anderson, D. L.: Theoretical basis of some empirical relations in seismology, B. Seismol. Soc. Am., 65, 10731095, 1975.

Kiratzi, A. A., Wagner, G. S., and Langston, C. A.: Source Parameters of Some Large Earthquakes in Nortern Aegean Determined by Body Waveform Inversion, Pure Appl. Geophys., 135, 515527, 1991.

Kiratzi, A. A., Karakaisis, G. F., Papadimitriou, E. E., and Papazachos, B. C.: Seismic-Source Parameter Relations for Earthquakes in Greece, Pure Appl. Geophys., 123, 27-41, 1985.

Kostopoulos, D., Varotsos, P., and Mourikis, S.: Conductivity of crystalline NaI, Can. J. Phys., 53, 1318-1320, 1975.

Le Pichon, X., Lyberis, N., and Alvarez, F.: Subsidence history of North Aegean through, in: The geological evolution of the Eastern Mediterranean, edited by: Dixon, J. E. and Robertson, A. H., Blackwell Scientif. Pub., Oxford, Geol. Soc. London, Sp. Publ. No. 17, 727-741, 1984.

Papazachos, B., Kiratzi, A., Karakostas, B., Panagiotopoulos, D., Scordilis, E., and Mountrakis, D.: Surface Fault Traces, Fault Plane Solution and Spatial Distribution of the Aftershocks of the September 13, 1986 Earthquake of Kalamata (Southern Greece), Pure Appl. Geophys., 126, 55-68, 1988.

Sarlis, N., Lazaridou,M., Kapiris, P., and Varotsos, P.: Numerical model of the selectivity effect and the $\Delta \mathrm{V} / \mathrm{L}$ criterion, Geophys. Res. Lett., 26, 3245-3248, 1999.
Sornette, D., Lagier, M., Roux, S., and Hansen, A.: Critical piezoelectricity in percolation, J. Phys. France, 50, 2201-2216, 1989.

Stavrakakis, G. and Blionas, S.: Eastern Mediterranean Region Based on an Iterative Maximum Entropy Technique, Pure Appl. Geophys., 132, 679-698, 1990.

Stavrakakis, G., Chouliaras, G., and Panopoulou, G.: Seismic Source Parameters for the ML=5.4 Athens Earthquake (7 September 1999) from a New Telemetric Broad Band Seismological Network in Greece, Nat. Hazards, 27, 47-60, 2002.

Surkov, V., Uyeda, S., Tanaka, H., and Hayakawa, M.: Fractal Properties of medium and seismoelectric phenomena, J. Geodynamics, 33, 477-487, 2002.

Varotsos, P.: Comments on the formation entropy of a Frenkel defect in BaF2 and CaF2, Phys. Rev. B-Solid St., 13, p. 938, 1976.

Varotsos, P. A.: Temperature and pressure dependence of defect formation volume in ionic crystals, J. Phys. Lett.-Paris, 38, L455L458, 1977.

Varotsos, P.: On the temperature variation of the bulk modulus of mixed alkali halides, Phys. Status Solidi-B, 99, K93-K96, 1980.

Varotsos, P. A.: The Physics of Seismic Electric Signals, TerraPub, Tokyo, 2005.

Varotsos, P.: Comparison of models that interconnect point defect parameters, J. Appl. Phys., 101, article 123503, 8 pp., 2007.

Varotsos, P. A. and Mourikis, S.: Difference in conductivity between LiD and LiH crystals, Phys. Rev. B, 10, 5220-5224, 1974.

Varotsos, P. and Miliotis, D.: New aspects on the dielectric properties of the alkali halides with divalent impurities, J. Phys. Chem. Solids, 35, 927-930, 1974.

Varotsos, P. and Alexopoulos, K.: Estimation of the migration enthalpy and entropy for cation vacancy motion in alkali halides with the NaCl-type structure, Phys. Rev. B, 15, 2348-2351, 1977.

Varotsos, P. and Alexopoulos, K.: The curvature in conductivity plots of silver halides as a consequence of anharmonicity, J. Phys. Chem. Solids, 39, 759-761, 1978.

Varotsos, P. and Alexopoulos, K.: On the possibility of the enthalpy of a Schottky defect decreasing with increasing temperature, J. Phys. C-Solid State, 12, L761-L764, 1979.

Varotsos, P. and Alexopoulos, K.: Negative activation volumes of defects in solids, Phys. Rev. B, 21, 4898-4899, 1980.

Varotsos, P. and Alexopoulos, K.: Decisive importance of the bulk modulus and the anharmonicity in the calculation of migration and formation volumes, Phys. Rev. B, 24, 904-910, 1981.

Varotsos, P. and Alexopoulos, K.: Current methods of lattice defect analysis using dilatometry and self diffusion. Critical Review and proposals, Phys. Stat. Solidi B, 110, 9-31, 1982.

Varotsos, P. and Alexopoulos, K. : Physical properties of the variations of the electric field of the earth preceding earthquakes, I, Tectonophysics, 110, 73-98, 1984a.

Varotsos, P. and Alexopoulos, K.: Physical properties of the variations of the electric field of the earth preceding earthquakes, II. Determination of epicentre and magnitude, Tectonophysics, 110, 99-125, 1984b.

Varotsos, P. and Alexopoulos, K.: Connection between the formation volume and formation Gibbs energy in noble gas solids, Phys. Rev. B, 30, 7305-7306, 1984c.

Varotsos, P. and Alexopoulos, K.: Thermodynamics of Point Defects and their Relation with the Bulk Properties, North Holland, Amsterdam, 1986. 
Varotsos, P. and Lazaridou, M.: Latest aspects of earthquake prediction in Greece based on seismic electric signals, I, Tectonophysics, 188, 321-347, 1991.

Varotsos, P., Ludwig W. and Alexopoulos, K.: Calculation of the formation volume of vacancies in solids, Phys. Rev. B, 18, $2683-$ 2691, 1978.

Varotsos, P., Alexopoulos, K., and Lazaridou M.: Latest aspects of earthquake earthquake prediction in Greece based on Seismic Electric Signals II, Tectonophysics, 224, 1-37, 1993.

Varotsos, P. A., Sarlis, N. V., and Skordas, E. S.: Long-range correlations in the electric signals that precede rupture, Phys. Rev. E, 66, article 011902, 7 pp., 2002.

Varotsos, P. A., Sarlis, N. V., and Skordas, E. S.: Long-range correlations in the electric signals that precede rupture: further investigations, Phys. Rev. E, 67, article 021109, 13 pp., 2003a.

Varotsos, P., Sarlis, N., and Skordas, E.: Attempt to distinguish electric signals of a dichotomous nature, Phys. Rev. E, 68, article 031106, 7 pp., 2003b.

Varotsos, P. A., Sarlis, N. V., and Skordas, E. S.: Detrended fluctuation analysis of the magnetic and electric field variations that precede rupture, CHAOS, 19, article 023114, arXiv:cond mat: 0904.2465v8, 7 pp., 14 November 2009a.

Varotsos, P., Alexopoulos, K., Lazaridou, M., and Nagao, T.: Earthquake predictions issued in Greece by seismic electric signals since February 6, 1990, Tectonophysics, 224, 269-288, 1993.
Varotsos, P., Eftaxias, K., Vallianatos, F., and Lazaridou, M.: Basic principles for evaluating an earthquake prediction method, Geophys. Res. Lett., 23, 1295-1298, 1996.

Varotsos, P., Sarlis, N., Lazaridou, M., and Kapiris, P.: Transmission of stress induced electric signals in dielectric media, J. Appl. Phys., 83, 60-70, 1998.

Varotsos, P. A., Sarlis, N. V., Skordas, E. S., and Lazaridou, M.: Natural entropy fluctuations discriminate similar looking electric signals emitted from systems of different dynamics, Phys. Rev. E, 71, article 011110, 11 pp., 2005.

Varotsos, P. A., Sarlis, N. V., Skordas, E. S., and Lazaridou, M. S.: The fluctuations, under time reversal, of the natural time and the entropy distinguish similar looking electric signals of different dynamics, J. Appl. Phys., 103, article 014906, 12 pp., 2008; arXiv:cond mat: 0707.3074v3, 5 February 2009, arXiv:cond mat: 0707.3074v4, 20 February 2009b.

Varotsos, P., Sarlis, N., Skordas, E., Tanaka, H., and Lazaridou, M.: Entropy of seismic electric signals: analysis in natural time under time reversal, Phys. Rev. E, 73, article 031114, 8 pp., 2006 a.

Varotsos, P., Sarlis, N., Skordas, E., Tanaka, H., and Lazaridou, M.: Attempt to distinguish long-range temporal correlations from the statistics of the increments by natural time analysis, Phys. Rev. E, 74, article 021123, 12 pp., 2006b. 